\title{
Quantum Synchronization of a Driven Self-Sustained Oscillator
}

\begin{abstract}
Stefan Walter, Andreas Nunnenkamp, and Christoph Bruder
Department of Physics, University of Basel, Klingelbergstrasse 82, CH-4056 Basel, Switzerland

(Received 26 July 2013; revised manuscript received 16 October 2013; published 6 March 2014)

Synchronization is a universal phenomenon that is important both in fundamental studies and in technical applications. Here we investigate synchronization in the simplest quantum-mechanical scenario possible, i.e., a quantum-mechanical self-sustained oscillator coupled to an external harmonic drive. Using the power spectrum we analyze synchronization in terms of frequency entrainment and frequency locking in close analogy to the classical case. We show that there is a steplike crossover to a synchronized state as a function of the driving strength. In contrast to the classical case, there is a finite threshold value in driving. Quantum noise reduces the synchronized region and leads to a deviation from strict frequency locking.
\end{abstract}

DOI: 10.1103/PhysRevLett.112.094102

PACS numbers: 05.45.Xt, 42.65.-k, 07.10.Cm

Synchronization is an intriguing phenomenon exhibited by a wide range of physical, chemical, and biological systems [1]. The basic setting consists of coupled selfoscillating systems synchronizing their motion; examples include such different phenomena as orbital resonances in planetary motion or the rhythm of muscle cells in mammal hearts. A paradigmatic and widely studied model of synchronization is the Kuramoto model of coupled limitcycle oscillators $[2,3]$.

The most fundamental scenario of classical synchronization is the frequency locking of a self-sustained oscillator which is externally driven by a harmonic force $[1,4]$. A selfsustained oscillator takes energy from a source, e.g., by negative damping, and can therefore maintain stable oscillatory motion and an undetermined phase in the presence of dissipation. If the oscillator is additionally driven by a harmonic force, there is a finite range of detuning for which the oscillator is frequency locked to the drive, and noise can reduce or destroy this range of synchronization [1]. There are also regimes of frequency entrainment in which the oscillator frequency is pulled towards the drive frequency, but does not reach it. In this case, the frequency of the driven oscillator, the observed frequency $\omega_{\mathrm{obs}}$, differs from both the natural frequency of the oscillator and the drive frequency. The simplest model exhibiting these effects is the Van der Pol oscillator [1] which allows the analysis of the complex phenomenology of synchronization.

Recently, the question if synchronization exists in quantum systems has attracted a lot of interest. There have been important first attempts to address this problem theoretically, from communities as diverse as trapped atomic ensembles, Josephson junctions, and nanomechanical systems [5-15]. Optomechanical systems [16] appear to offer a particularly promising approach. Recent experiments have reported classical synchronization of nanomechanical oscillators $[17,18]$, the quantum many-body dynamics of an array of identical optomechanical cells has been predicted to show synchronized behavior [8], and quantitative measures for quantum synchronization based on the Heisenberg uncertainty principle have been applied to two and many coupled optomechanical cells [9].

In this Letter, we analyze the most basic example of quantum synchronization: a quantum version of the harmonically driven Van der Pol oscillator. To complement other recent work which studied phase locking [12], we focus on frequency entrainment and frequency locking for various detunings, driving strengths, and nonlinear damping rates. We establish the power spectrum as a theoretical and experimental tool to characterize the observed frequency. As one of our main results, we find a steplike crossover to a synchronized state as a function of the strength of the harmonic drive. In contrast to the classical case, there is a finite threshold value in driving. We find that quantum noise reduces the synchronized region as compared to the (noiseless) classical case and leads to a deviation from strict frequency locking. For weak (strong) driving and for small nonlinear damping rates, frequency entrainment is reduced (enhanced) by increasing the nonlinear damping rate. Finally, we present a realization of our model in an optomechanical setup demonstrating that our study is directly relevant to current experimental work.

Model.-We numerically analyze a quantum version of the Van der Pol oscillator subject to an external harmonic drive. The master equation for this model reads (in the frame rotating with the external drive)

$$
\begin{aligned}
\frac{d \rho}{d t}= & -i\left[-\Delta \hat{b}^{\dagger} \hat{b}+i \Omega\left(\hat{b}-\hat{b}^{\dagger}\right), \rho\right]+\gamma_{1} \mathcal{D}\left[\hat{b}^{\dagger}\right] \rho \\
& +\gamma_{2} \mathcal{D}\left[\hat{b}^{2}\right] \rho
\end{aligned}
$$

where $\Delta$ is the detuning of the drive frequency with respect to the natural frequency of the undriven oscillator, $\Omega$ determines the strength of the external drive, and we have set $\hbar=1$. The coefficients $\gamma_{1}$ and $\gamma_{2}$ describe negative and 
nonlinear damping, respectively, and $\mathcal{D}$ are Lindblad dissipators, $\mathcal{D}[O] \rho=O \rho O^{\dagger}-(1 / 2)\left\{O^{\dagger} O, \rho\right\}$. We numerically calculate the steady-state solution $\rho_{s s}$ of the master equation Eq. (1) from which we obtain the steady-state Wigner function $W_{s s}(x, p)=(1 / \pi) \int d y \exp (-2 i p y)\langle x+y$ $\left.\left|\rho_{s s}\right| x-y\right\rangle$.

The corresponding equation of motion for $\beta=\langle\hat{b}\rangle$,

$$
\frac{d}{d t} \beta=i \Delta \beta+\frac{\gamma_{1}}{2} \beta-\gamma_{2}|\beta|^{2} \beta-\Omega,
$$

is the equation of motion for the classical Van der Pol oscillator. Separating the dynamics of the complex variable $\beta=r e^{i \phi}$ into the dynamics of amplitude $r$ and phase $\phi$ leads to the classical amplitude equation $d r / d t=\left(\gamma_{1} / 2-\right.$ $\left.\gamma_{2} r^{2}\right) r-\Omega \cos \phi$ and $d \phi / d t=\Delta+(\Omega / r) \sin \phi$ which is called the Adler equation [4] if the amplitude dynamics are negligible $(\dot{r}=0)$.

Synchronization regimes. - The synchronization regimes of the classical driven Van der Pol oscillator can be determined from the phase-space trajectories of its latetime dynamics; for details on synchronization regimes and a phase diagram of (classical) synchronization see [19]. To characterize phase locking in the system we investigate the probability histogram of the phase, $P(\phi)$. In the classical case, $P_{\mathrm{cl}}(\phi)$ can be obtained from the late-time phase-space trajectory. In the quantum case, we use the Wigner function, a quasiprobability distribution for the canonically conjugate operators $\hat{x}=x_{\mathrm{zpf}}\left(\hat{b}+\hat{b}^{\dagger}\right)$ and $\hat{p}=-i p_{\mathrm{zpf}}$ $\left(\hat{b}-\hat{b}^{\dagger}\right)$, as an analog of the classical phase space density, where $x_{\mathrm{zpf}}=1 / \sqrt{2 m \omega_{0}}$ is the amplitude of the zero-point fluctuations of the oscillator with natural frequency $\omega_{0}$, and similarly $p_{\text {zpf }}=\sqrt{m \omega_{0} / 2}$. Here, $P_{\mathrm{qm}}(\phi)$ can be calculated from $W_{s s}(x, p)$ by transforming from the variables $x$ and $p$ to radius and phase and integrating over the radius. In Figs. 1 and 2 we show the classical phase-space trajectory, the Wigner function, and the corresponding probability histograms for the phase variable. In both figures, we decrease the detuning at fixed driving strength.
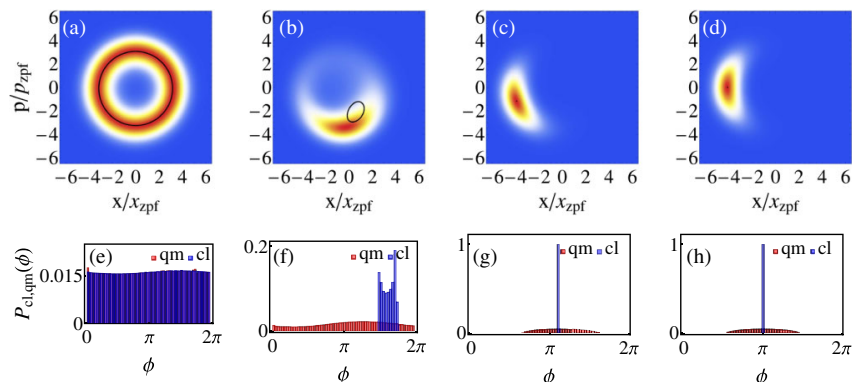

$\mathrm{x} / x_{\mathrm{zp}}$
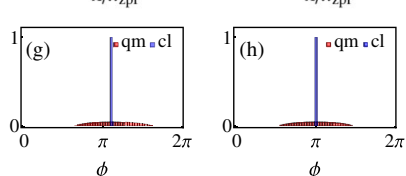

FIG. 1 (color online). Classical phase-space trajectory (black solid line), Wigner function, and the corresponding phase probability histograms for $\Omega / \gamma_{1}=1$ and $\gamma_{2} / \gamma_{1}=0.1$. (a),(e) $\Delta / \gamma_{1}=16, \quad$ (b),(f) $\Delta / \gamma_{1}=0.6, \quad$ (c),(g) $\Delta / \gamma_{1}=0.1$, and (d),(h) $\Delta / \gamma_{1}=0$.
In the classical case, we observe phase locking, as indicated by the $\delta$-function-like shape of $P_{\mathrm{cl}}(\phi)$ in $(\mathrm{g})$, (h), whereas in the quantum case $P_{\mathrm{qm}}(\phi)$ is smeared out even in this regime due to quantum noise. In the quantum case, a trend towards synchronization of the quantum oscillator is visible since the expectation values $\langle\hat{x}\rangle$ and $\langle\hat{p}\rangle$ become finite with smaller $\Delta$; see Figs. 1(d) and 2(d) where the Wigner function concentrates in a displaced blob.

The difference between the cases $\gamma_{2}<\gamma_{1}$ (Fig. 2) and $\gamma_{2}>\gamma_{1}$ (Fig. 1) is most prominent for large detuning $\Delta$. For $\gamma_{2}<\gamma_{1}$ the Wigner function is of ring shape and the classical trajectory almost coincides with the maximum of the Wigner function, Fig. 2(a). For $\gamma_{2}>\gamma_{1}$, however, the maximum of the Wigner function and the classical trajectory no longer coincide. In this case, the quantum nature (i.e., the discrete level structure) of the self-sustained oscillator becomes more important. In the limit $\gamma_{2}>\gamma_{1}$ the rate at which the oscillator loses two phonons dominates over the rate at which it gains one phonon; see Eq. (1). Thus, in the steady state for a small and moderate driving strength only the lowest Fock states of the mode $\hat{b}$ are occupied.

Spectra and observed frequency.-To investigate frequency entrainment and locking we use the classical and quantum-mechanical power spectrum. In the classical case, it reads

$$
S_{\mathrm{cl}}(\omega)=\int_{-\infty}^{\infty} d t e^{i \omega t} \overline{\beta^{*}(t) \beta(0)},
$$

where the bar denotes time averaging. In the quantum case, we consider the spectrum

$$
S_{\mathrm{qm}}(\omega)=\int_{-\infty}^{\infty} d t e^{i \omega t}\left\langle\hat{b}^{\dagger}(t) \hat{b}(0)\right\rangle,
$$

where $\langle\cdot\rangle$ denotes the average with respect to the full quantum-mechanical density matrix.

Figure 3 shows typical spectra. The classical spectrum has a $\delta$-shaped peak at the observed frequency $\omega_{\text {obs }}$ (in the frame rotating with the drive). In addition to this main peak,
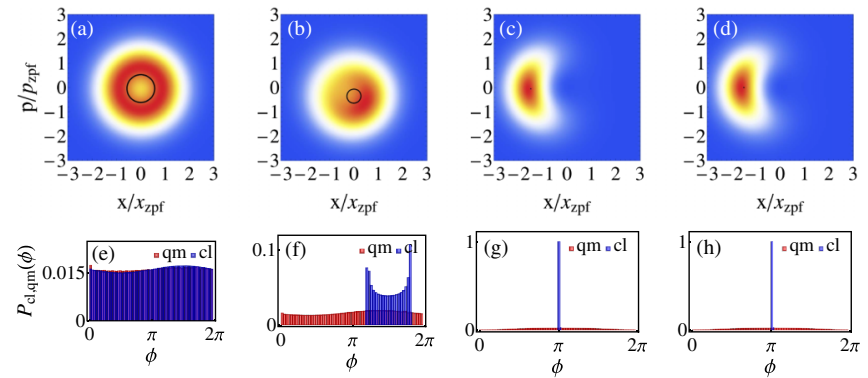

FIG. 2 (color online). Classical phase-space trajectory (black solid line), Wigner function, and the corresponding phase probability histograms for $\Omega / \gamma_{1}=3$ and $\gamma_{2} / \gamma_{1}=3$. (a),(e) $\Delta / \gamma_{1}=100$, (b),(f) $\Delta / \gamma_{1}=12$, (c),(g) $\Delta / \gamma_{1}=0.1$, and (d),(h) $\Delta / \gamma_{1}=0$. 
satellite peaks emerge at higher harmonics of the main frequency [1] (not shown in Fig. 3). In the quantum case, the spectrum is not an even function of frequency, and the peak has finite width. In the classical and the quantum case, the observed frequency can be obtained from the position of the maximum of $S_{\mathrm{cl}, \mathrm{qm}}(\omega)$ if the peaks are well separated. The spectra are thus a convenient tool to study synchronization properties, and we will use them in the following to analyze the dependence of $\omega_{\mathrm{obs}}$ on the detuning $\Delta$ and the driving strength $\Omega$.

Synchronization.-To investigate synchronization we plot in Figs. 4(a) and 4(b) the observed frequency $\omega_{\text {obs }}$ as a function of detuning $\Delta$ for fixed driving strengths $\Omega$ and compare the classical case with the quantum case for $\gamma_{2}<\gamma_{1}$.

Figure 4(a) shows that for weak driving the classical oscillator is frequency locked to the drive over a finite range of $\Delta$. In the quantum-mechanical model, however, there is only weak frequency entrainment (cf. inset), except at $\Delta=0$ where the frequency of the oscillator trivially agrees with the drive. This is in stark contrast to the classical case, where an arbitrarily weak drive leads to a small, but finite synchronized region. At a larger driving strength, strong frequency entrainment and a tendency towards frequency locking for the quantum model can be seen in Fig. 4(b). However, as the inset shows, there is no exact frequency locking at finite detuning $\Delta \neq 0$. We attribute the absence of frequency locking to quantum noise, since for the classical Van der Pol oscillator, noise can reduce or even destroy the synchronization region [1]. A semiclassical approach valid in the limit $\gamma_{1} \gg \gamma_{2}$ yields the appropriate (external) white noise which mimics the (intrinsic) quantum noise and can be used to compare the quantum case to a noisy classical Van der Pol oscillator [12].

To analyze the synchronization behavior in more detail, we now fix the detuning $\Delta$ and study the dependence of

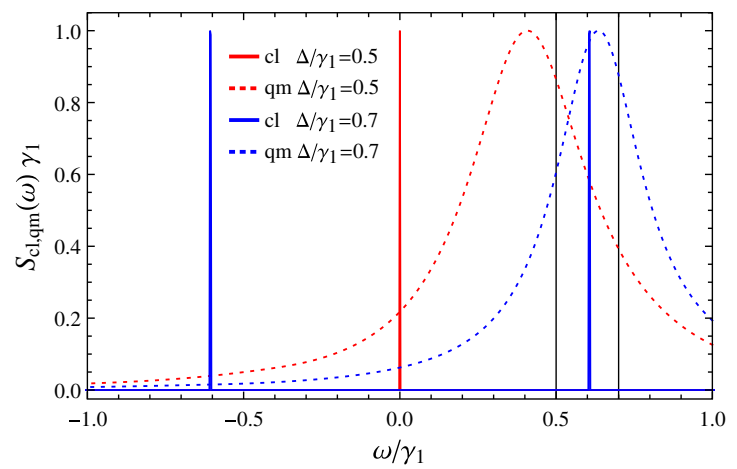

FIG. 3 (color online). Normalized classical (full lines) and quantum (dashed lines) spectrum. For $\Delta / \gamma_{1}=0.7$ (blue) both the quantum and classical model show a peak at a frequency smaller than the detuning (indicated by vertical black lines). For $\Delta / \gamma_{1}=$ 0.5 (red) the classical model is frequency locked to the drive, while the quantum model is not. The other parameters are $\Omega / \gamma_{1}=1$ and $\gamma_{2} / \gamma_{1}=0.1$. $\omega_{\text {obs }}$ on the driving strength $\Omega$ and the nonlinear damping rate $\gamma_{2}$.

Figure 5 shows $\omega_{\text {obs }}\left(\Omega / \gamma_{1}\right)$ for different damping rates $\gamma_{2} / \gamma_{1}$. A weak drive leads to a small amount of frequency entrainment. However, if the drive exceeds a threshold whose position depends on the nonlinear damping $\gamma_{2} / \gamma_{1}$, a steplike crossover to strong frequency entrainment occurs. Moreover, the influence of $\gamma_{2}$ is different below and above the threshold. For weak driving, increasing $\gamma_{2} / \gamma_{1}$ (and thereby increasing the quantum nature of the oscillator) reduces frequency entrainment; i.e., for small driving a large value of $\gamma_{2} / \gamma_{1}$ inhibits synchronization. For large driving, the situation is opposite: increasing the nonlinear damping rate $\gamma_{2}$ supports frequency entrainment (see right inset of Fig. 5). To investigate the case of large $\gamma_{2} / \gamma_{1}$ in more detail, we solve Eq. (1) perturbatively. In the limit $\gamma_{2} / \gamma_{1} \rightarrow \infty$ and for $\Omega / \gamma_{1}=\Delta / \gamma_{1}=0$ the steady state of Eq. (1) can be found: $\rho_{s s} \rightarrow \frac{2}{3}|0\rangle\left\langle 0\left|+\frac{1}{3}\right| 1\right\rangle\langle 1|$. Including a finite drive and detuning leads to nonzero off-diagonal matrix elements $\left\langle 1\left|\rho_{s s}\right| 0\right\rangle$ and $\left\langle 0\left|\rho_{s s}\right| 1\right\rangle$. In this case, making use of the quantum regression theorem, we obtain an analytic expression (too lengthy to be displayed here) for the spectrum $S_{\mathrm{qm}}(\omega)$ which is independent of $\gamma_{2}$.
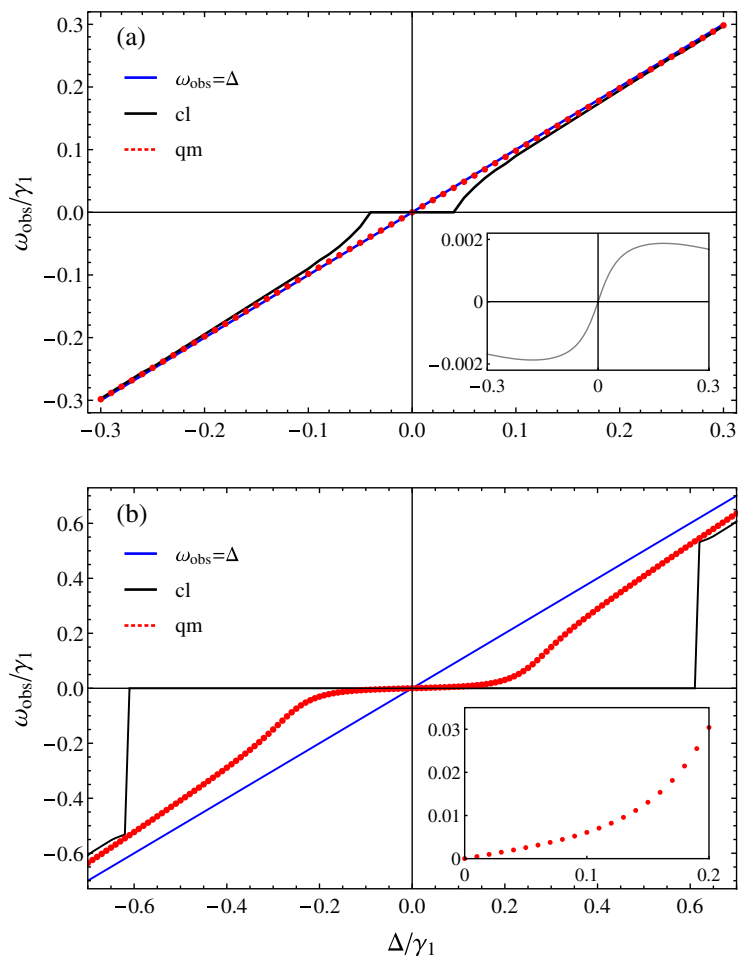

FIG. 4 (color online). Observed frequency $\omega_{\text {obs }}$ vs detuning $\Delta$ for $\gamma_{2} / \gamma_{1}=0.1$. Blue: undriven case. Black: classical model. Red: quantum model. In (a), for $\Omega / \gamma_{1}=0.1$, the quantum model shows weak frequency entrainment. Inset: difference between undriven and the quantum mechanical case. In (b), for $\Omega / \gamma_{1}=1$, the quantum model shows strong frequency entrainment and a tendency towards frequency locking. Inset: zoom-in for the quantum case at small $\Delta$. 


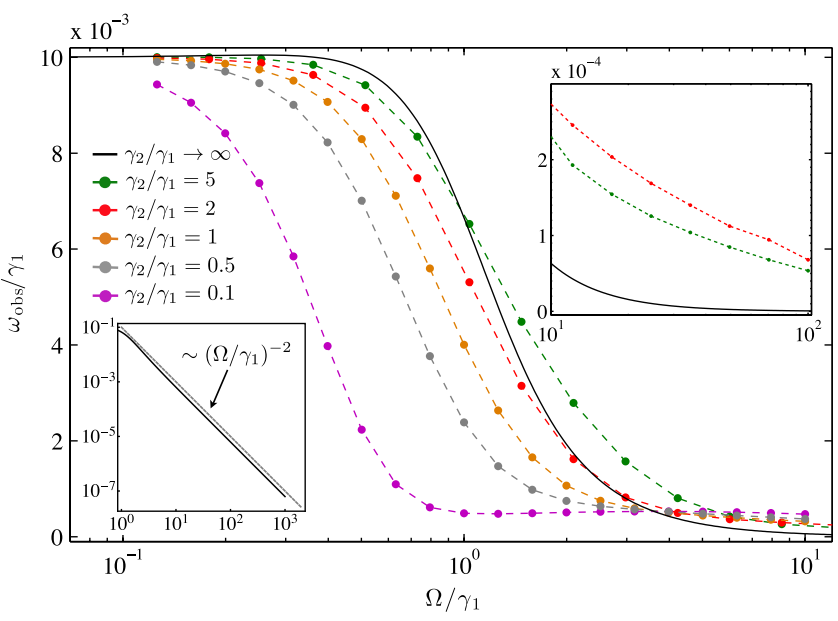

FIG. 5 (color online). Observed frequency $\omega_{\text {obs }}$ vs driving $\Omega$ in the quantum model for various values of $\gamma_{2} / \gamma_{1}$ and $\Delta / \gamma_{1}=0.01$. A steplike crossover from weak to strong entrainment can be observed. At larger $\gamma_{2} / \gamma_{1}$ the step shifts to larger driving strengths. Below threshold, increasing $\gamma_{2} / \gamma_{1}$ reduces frequency entrainment, i.e., inhibits synchronization. Right inset: Observed frequency at larger driving. Increasing $\gamma_{2} / \gamma_{1}$ leads to increased frequency entrainment, i.e., improved synchronization. Left inset: The observed frequency $\omega_{\mathrm{obs}}$ for $\gamma_{2} / \gamma_{1} \rightarrow \infty$ (black line) vanishes like $\left(\Omega / \gamma_{1}\right)^{-2}$ (gray dashed line).

The analytic result for the observed frequency is also shown in Fig. 5. Although we do not find genuine frequency locking in the quantum case, the difference between observed frequency and drive frequency can be made arbitrarily small. Our analytical treatment predicts the observed frequency to vanish like $\left(\Omega / \gamma_{1}\right)^{-2}$ (see left inset of Fig. 5).

To analyze the dependence on the nonlinear damping rate, Fig. 6 shows $\omega_{\text {obs }}\left(\gamma_{2} / \gamma_{1}\right)$ for different driving strengths $\Omega$. Without driving there is no entrainment and the observed frequency coincides with the detuning (black line). Driving the oscillator stronger generally enhances frequency entrainment. However, in the left part of Fig. 6, i.e., for $\gamma_{2} / \gamma_{1} \lesssim 1$, there is an interesting difference in the dependence on $\gamma_{2} / \gamma_{1}$. For weak driving, frequency entrainment is reduced by increasing $\gamma_{2} / \gamma_{1}$. For strong driving, on the other hand, increasing $\gamma_{2} / \gamma_{1}$ enhances frequency entrainment and synchronization (lower three curves). In the limiting case $\gamma_{2} / \gamma_{1} \rightarrow \infty, \omega_{\text {obs }}$ becomes independent of $\gamma_{2}$. The observed frequency obtained from our analytical solution is shown at the very right of Fig. 6.

Realization.-Optomechanical systems are suitable candidates to study synchronization. We therefore describe a possible scheme to realize the dynamics given by Eq. (1) in an optomechanical system. The two dissipative processes $\gamma_{1} \mathcal{D}\left[\hat{b}^{\dagger}\right]$ and $\gamma_{2} \mathcal{D}\left[\hat{b}^{2}\right]$ can readily be engineered in a "membrane-in-the-middle" optomechanical setup [20]. Such a setup gives rise to a quadratic optomechanical coupling which can be used to favor two-phonon absorption and emission [21]. The dissipative terms can be created

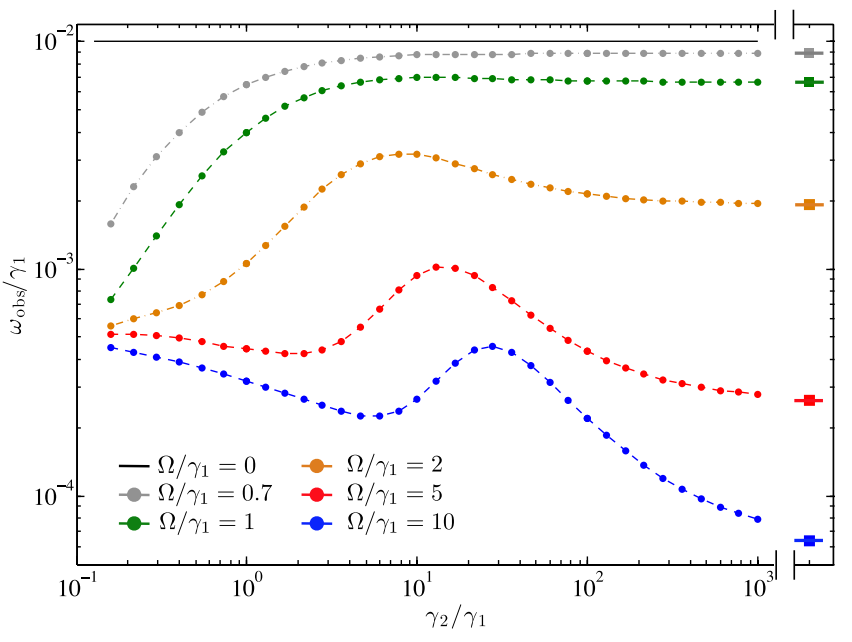

FIG. 6 (color online). Observed frequency $\omega_{\text {obs }}$ vs nonlinear damping rate $\gamma_{2}$ in the quantum model for various driving strengths $\Omega$ and $\Delta / \gamma_{1}=0.01$. Increasing the driving strength is seen to enhance frequency entrainment. For weak (strong) driving and at $\gamma_{2} / \gamma_{1} \lesssim 1$, frequency entrainment is reduced (enhanced) by increasing $\gamma_{2} / \gamma_{1}$. Squares on the right are obtained analytically in the limit $\gamma_{2} / \gamma_{1} \rightarrow \infty$.

using two lasers. The first laser is detuned to the blue one-phonon sideband and gives rise to the negative damping term $\sim \gamma_{1}$. The second laser is detuned to the red two-phonon sideband leading to the nonlinear damping $\sim \gamma_{2}$. Synchronization can be probed by changing the frequency of the harmonic drive and observing the spectrum of the mechanical oscillator. A different strategy based on trapped ions to realize the dynamics of the Van der Pol oscillator in a quantum system was put forward in Ref. [12].

Conclusion.-We have analyzed the quantum Van der Pol oscillator in the presence of an external harmonic drive. Our study establishes the power spectrum, that is readily accessible in current nanomechanical experiments, as an important observable of quantum synchronization. The steady-state Wigner function can indicate a synchronized state, but it is not sufficient to obtain the observed frequency and thus does not allow for studying frequency entrainment and its locking. The information contained in the power spectrum thus neatly complements previous analyses of phase locking. We have shown the existence of weak and strong frequency entrainment in the quantum case and found a threshold in the strength of the drive below which there is only weak frequency entrainment between the oscillator and the drive. Our study contributes new insights in understanding synchronization in the quantum regime and also allows various extensions as, for instance, the investigation of synchronization of two (or an ensemble of) nonidentical quantum Van der Pol oscillators. Another interesting question is whether engineered noise [22,23] (classical and/or quantum) can favor synchronization. 
We would like to acknowledge stimulating discussions with S. Aldana and F. Marquardt. This work was financially supported by the Swiss SNF and the NCCR Quantum Science and Technology.

Note added.-Just before submitting this manuscript we became aware of the work of Lee and Sadeghpour [12] studying a quantum Van der Pol oscillator with a resonant weak drive.

[1] A. S. Pikovsky, M. Rosenblum, and J. Kurths, Synchronization: A Universal Concept in Nonlinear Science (Cambridge University Press, New York, 2001).

[2] Y. Kuramoto, Prog. Theor. Phys. Suppl. 79, 223 (1984).

[3] J. A. Acebrón, L. L. Bonilla, C. J. Pérez Vicente, F. Ritort, and R. Spigler, Rev. Mod. Phys. 77, 137 (2005).

[4] R. Adler, Proc. IRE 34, 351 (1946); R. Adler reprinted as Proc. IEEE 61, 1380 (1973).

[5] O. V. Zhirov and D. L. Shepelyansky, Eur. Phys. J. D 38, 375 (2006).

[6] I. Goychuk, J. Casado-Pascual, M. Morillo, J. Lehmann, and P. Hänggi, Phys. Rev. Lett. 97, 210601 (2006).

[7] G. Heinrich, M. Ludwig, J. Qian, B. Kubala, and F. Marquardt, Phys. Rev. Lett. 107, 043603 (2011).

[8] M. Ludwig and F. Marquardt, Phys. Rev. Lett. 111, 073603 (2013).

[9] A. Mari, A. Farace, N. Didier, V. Giovannetti, and R. Fazio, Phys. Rev. Lett. 111, 103605 (2013).

[10] T.E. Lee and M. C. Cross, Phys. Rev. A 88, 013834 (2013).
[11] A. M. Hriscu and Yu. V. Nazarov, Phys. Rev. Lett. 110, 097002 (2013).

[12] T. E. Lee and H. R. Sadeghpour, Phys. Rev. Lett. 111, 234101 (2013).

[13] G. L. Giorgi, F. Galve, G. Manzano, P. Colet, and R. Zambrini, Phys. Rev. A 85, 052101 (2012).

[14] G. Manzano, F. Galve, G. L. Giorgi, E. Hernandez-Garcia, and R. Zambrini, Sci. Rep. 3, 1439 (2013).

[15] M. Xu, D. A. Tieri, E. C. Fine, J. K. Thompson, and M. J. Holland, arXiv:1307.5891 (2013).

[16] M. Aspelmeyer, T. J. Kippenberg, and F. Marquardt, arXiv:1303.0733 (2013).

[17] M. Zhang, G. S. Wiederhecker, S. Manipatruni, A. Barnard, P. McEuen, and M. Lipson, Phys. Rev. Lett. 109, 233906 (2012).

[18] M. H. Matheny, M. Grau, L. G. Villanueva, R. B. Karabalin, M. C. Cross, and M. L. Roukes, Phys. Rev. Lett. 112, 014101 (2014).

[19] See Supplemental Material at http://link.aps.org/ supplemental/10.1103/PhysRevLett.112.094102 for details on synchronization regimes and a phase diagram of (classical) synchronization.

[20] J. D. Thompson, B. M. Zwickl, A. M. Jayich, F. Marquardt, S. M. Girvin, and J. G. E. Harris, Nature (London) 452, 72 (2008).

[21] A. Nunnenkamp, K. Børkje, J. G. E. Harris, and S. M. Girvin, Phys. Rev. A 82, 021806(R) (2010).

[22] S. Diehl, A. Micheli, A. Kantian, B. Kraus, H. P. Büchler, and P. Zoller, Nat. Phys. 4, 878 (2008).

[23] F. Verstraete, M. M. Wolf, and J. I. Cirac, Nat. Phys. 5, 633 (2009). 